DOI: doi.org/10.21009/IJLECR.042.12

Received: 5 June 2018

Revised: 10 June 2018

Accepted: 14 August 2018

Published: 31 December 2018

\title{
CHARACTER BUILDING OF ETHICS IN UNDERGRADUATE ENGLISH CURRICULUM DOCUMENT
}

\author{
Mu'man $^{1, a)}$, Sri Sumarni ${ }^{1, b)}$, Ratna Dewanti ${ }^{1, c)}$
}

English Language Education Master Program, Faculty of Language and Arts State University of Jakarta ${ }^{1)}$

muman_pb16s2@mahasiswa.unj.ac.id ${ }^{\text {a) }}$,Sri.sumarni@unj.ac.id ${ }^{\text {b) }}$, rdewanti@unj.ac.id ${ }^{\text {) }}$,

\begin{abstract}
This study aimed to describe character building of ethics addressed in the undergraduate English curriculum documents. Curriculum document is a planning, implementation, evaluation, management, and administration of education programs either in written forms, spoken statements, descriptions, or archaeologies. The curriculum document which had been analyzed consisted of semester planning of teaching and learning program (SPTLP)/syllabus, learning material and assessment taken from three universities - one was from Jakarta and the others were from Bandung. This study used qualitative content analysis method. The result of this study showed that ethical values such as trustworthiness, respect, responsibility, fairness, caring, and citizenship were addressed in SPTLP/syllabus, learning materials, and assessments in each university either explicitly or implicitly in the form of words, phrases, and clauses. In SPTLP/syllabus, ethical values were presented in basic competences, learning objectives, and course policies. In learning materials, ethical values were presented in topics and contents while in assessments ethical values were presented in assessment competences, assessment criteria and assessment form, and course policies.
\end{abstract}

Keywords: Character Building, Ethics, and Curriculum Document.

Character building of ethics is an effort to build students' character in order to have moral values. In general, character building of ethics nowadays concerns not only to education fields but also to other fields such as in health, religion, business, communication, culture, therapy, and so forth. Therefore, every field must teach or require to insert minimally three ethical/moral studies such as either in the form of philosophy, ethics, or local wisdom (Syamsiyatun, 2013, p. 9). It is proved by previous studies about character building of ethics.

In educational field, ethics is meant as a belief, a moral responsibility, a set of moral value, and an honor code. As a belief, ethics is used as a reference to determine what is right and what is wrong. This ethics is used by academic staffs to assess and develop academic quality (Margetson, 1997, p. 128). As a moral responsibility, there 
are two meanings when ethics is related to a moral responsibility. First, ethics is meant as a guideline for students in order to have a responsibility. This moral responsibility is not only regulated to be performed by the students but also to be owned when they have been accomplished their study and entering the workplace (Spears, 2006). Second, ethics is meant as a guideline for the educators in engaging in communal processes to pursue the moral purposes of their work and address the ongoing challenges of daily work. This ethics is presented to unite educators in facing moral challenges among educators to educators, students to students, and educators to students (Furman, 2004).

Further, ethics is also meant as a set of moral value. There are two meanings when ethics is put as a set of moral value/moral value. First, ethics is used by academic community to filter ethical issues which enter to the university that can damage students' moral behavior because the ethics which enters to the university not only bring good morality but it also brings bad morality (Nijhof et al., 2012). Second, ethics is used by academic staffs to organize and manage students' activities including promoting ethical thinking for academic, career, and business purposes. By having a moral value, it will bring a good impact either for students personality or for academic (Safatly et al., 2017).

Next, ethics is meant as an honor code. The honor code here is a way to institutionalize ethics for the students to reduce academic cheating. There are two explanations when ethics is related to honor code - formal (explicit) and informal (implicit). Formal (explicit) is called as an explicit ethics institutionalization and informal (implicit) is called as an implicit ethics institutionalization (Popoola et al., 2017).

In other fields, ethics is meant as the study of good and bad values, the concepts and basic principles of appropriate human behaviors, a prescribed rules to guide behavior and the systematic guideline for shaping ethical behaviors. First, ethics is meant as the study of good and bad values. This ethics is applied in religion field as a guidance for the followers of certain religion to act and talk based on what have been taught by Prophets. For example, Islam always teaches to act and to talk like the prophet Muhammad SAW (Isyraqi, 2011).

Second, ethics is meant as the concepts and basic principles of appropriate human behaviors. This ethics is applied in business field as the regulation for all aspects - individual, company and also society. Individually, ethics is used to regulate a good relationship among employees. As a company, ethics is used to regulate a good relationship between employees and company and ethics is used to regulate a good relationship among company, societies, and customers (Sinaulan, 2016).

Third, ethics is meant as a prescribed rules to guide behavior. This ethics is used in communication field as a rule which have to be obeyed in communicating. Ethics here is rule to direct societies to use ethics in communication (Jovanovic \& Wood, 2006). Last, ethics is meant as the systematic guideline for shaping ethical behaviors. This ethics is used in nursing field. This ethics is addressed to the nurses so that they will have a value. This value concerns how the nurses put themselves personally as well as they put the patients (Butts, 2001).

From the ten researchers who have conducted the researches about ethics, the concepts and teachings of ethics either in educational fields or other fields are relatively the same. The difference is in application. Ethics in education field tends to be called as philosophically. It means that it is related to good and bad value. 
Meanwhile, ethics in other fields tends to be called as local wisdom where the application of this ethics is always related to one another.

As it has been discussed that ethics plays important roles in various fields, education places ethics as a basis for carrying out educational enterprises as well as a frame for educating students. It is intended to build, enact, and maintain ethics in education in other to be able to shape students' character to be ethical and have a moral value. Therefore, applying ethics in education filed not only gives examples in daily activities but ethics must also be written clearly in curriculum document like in semester planning of teaching and learning program (SPTLP) or syllabus, learning material, and assessment.

Building character of ethics becomes the concern of education in Indonesia and it is proved in the statement of National policy of building nation character in the plan of long-term national development year 2005-2025, in the regulation of Sistem Pendidikan Nasional (SISDIKNAS) no. 20 year 2003, in 2013 curriculum, and in the statement of Ministry of Education and Culture which requires to apply ethics - character building in education environment. This is pointed to every education institution from the basic education until higher education.

Even higher education does not escape the spotlight of the government in order to apply ethics in its curriculum document. It can be seen clearly in the Indonesian Qualification Frame of Reference. A requirement is mentioned in common description of attitude and value system. Students must have moral, ethics, and good personality. Even the curriculum of higher education is developed by each university, this must still refer to government regulation. Therefore, ethics must be presented in every higher education curriculum.

The desire of government to build a strong national character is very great. This can be seen since Indonesia became an independent country up to current government, character education is prioritized in education filed. This is clearly regulated in the law of 1945, in law No. 17 of 2007, in president rule No. 87 / 2017, in the character education stipulation of Ministry of Education and Culture year 2011, in the Indonesian Qualification Frame of Reference, in National Education System no. 20 year 2003, and in National Standard for Higher Education.

This government program needs a hard effort to achieve the target. This must involve family, environment, and education field. These three fields have a respective role. Education field usually becomes a public spotlight because education field is a place to shape students' character. It can be said that if students' character looks good, that education field is also good and if students' character looks bad, that education field is also bad.

It can be seen from the existing phenomena, education units have not applied education character yet as well especially in higher education field. A disrespectful behavior still occurs such as cheating friend' assignment, coming late, using inappropriate language, and so forth. These problems encourage the researcher to conduct the research about character education - ethics - which is addressed in curriculum documents.

In relevant to this study, some researchers have conducted the same theme. Such as analyzing students' character based on character education. The result showed that most of students have not applied akhlakul karimah well. They have just understood that ahlaqul karimah is important (Hidayat, 2013). Further, analyzing teacher personality and the effort of teacher in shaping students' character. The result was that teacher 
personality determines student's personality. It means that if teacher who has good personality will also create the students who have good personality. Personality here meant as ethics (Sri et al., 2013).

Then, analyzing character education policy including the implementation, constraint, and strategies in elementary school. The result was shown especially from academic document analysis that it was found in the learning syllabus which taught character education for students clearly (Acetylena, 2013). Besides, there was the analysis of emphasizing morals, values, ethics, and character education in science curriculum and science teaching. The result was found that morals, values, ethics, and character education must be in the same part and they cannot be separated (Chowdhuri, 2016). The last research was conducted to find out the most effective way to apply moral value in primary school. The result showed that the most effective way to apply moral value is that students are introduced to the important of moral value and guided to identify ethical value. (Wing, 2014).

From the previous researches, the researcher saw the gap left. The niche is the form of effort to find out character building of ethics in undergraduate English curriculum documents such as in semester planning of teaching and learning program (SPTLP)/syllabus, learning materials, and assessments taken only from English education major. Hence, this study aims to investigate character building of ethics in undergraduate English curriculum documents.

\section{METHOD}

This study used content analysis. Content analysis is the study of an indirect human behavior. The forms of human communication could be documents, beliefs, attitudes, values, and the ideas which is present when people are doing a communication. The five forms of that communication can be categorized into content analysis because those consist of human behavior. Hence, the analysis of communication content can give the information about how people live in this world (Fraenkel, 2009, p. 472).

There are seven steps in analyzing the data; 1) reviewing all available data which have been collected from each university, 2) selecting the data, 3) reducing the data according to the research questions, 4) compiling the data, 5) coding the data, 6) displaying the data in data analysis table, 7) verifying/concluding the data. In the stage of coding the data, the researcher did steps as the following; a) each university was marked with code A-C. State Islamic University of Sunan Gunung Djati Bandung was marked with code A., Nusantara Islamic University was marked with code B., and State University of Jakarta was marked with code C.

b) The three parts of document are marked with code A-C. SPTLP/syllabus was marked with code A, learning material was marked with code B, and assessment was marked with code C. c) each subjects selected from each university were marked with code 1.-7. d) Parts of data source was marked with code 1-3. Data description was marked with code a-h. This code was based on how many data covering the ethical values each subject has.

From some forms of written documents, the researcher analyzed curriculum documents - undergraduate English curriculum documents - with qualitative method. Qualitative method is used to collect and analyze the data in this research. In the process of collecting the data, the researcher did some steps to gather the data needed in accordance with the research questions and the process of analyzing the data was done 
through three steps including data reduction, data display, and conclusion/verification (Miles \& Huberman, 1994).

\section{RESULTS AND DISCUSSION}

In order to answer the three research questions about three documents analyzed from three different universities, the research question one will be based on the document analysis of semester planning of teaching and learning program (SPTLP)/syllabus. Research question two will be based on the document analysis of learning materials. Then, research question three will be based on the document analysis of assessments.

The totality of English subjects analyzed are 105 from three universities have been analyzed. Because there the similarity of data found in each subject, the researcher took one subject to become as the data source. Hence, the real data sources used are seven was taken from University A, seven was taken from University B, and six was taken from University C. In the study, the researcher made a different analysis among them.

From the result of analysis in SPTLP/syllabus, it has been found 11 data in University B addressed in basic competences from six English subjects, nine data in University B addressed in learning objectives from five English subjects, and seven data in University $\mathrm{C}$ addressed in learning objectives and course policies from five English subjects.

For the research question two asking about the addressing ethical values in learning materials, it has been found 13 data in University A in lesson topics from four English subjects, nine data in University B in lesson contents from two English subjects, and two data in University $\mathrm{C}$ from two English subjects. Then, the last is asking about ethical values addressed in assessments. There were four data in University A addressed in assessment competences from 43 the same English subjects, seven data found in University B addressed in assessment criteria and assessment format from six English subjects, and 6 data found in university $\mathrm{C}$ addressed in course policies from 28 the same English subjects. To see the data clearly, it can be seen in the table as the following.

Table: 1.1 Data Identification table of data findings from SPTLP/syllabus, Learning Materials, and Assessments

\begin{tabular}{|c|c|c|c|c|c|c|}
\hline \multirow{2}{*}{$\begin{array}{l}\text { R. } \\
\text { Q }\end{array}$} & \multirow{2}{*}{$\begin{array}{l}\text { Data } \\
\text { Sources }\end{array}$} & \multirow{2}{*}{$\begin{array}{l}\text { Parts of data } \\
\text { sources }\end{array}$} & \multirow[t]{2}{*}{ Data } & \multirow[b]{2}{*}{$\begin{array}{l}\text { Core ethical values } \\
\text { Proposed by Josephson } \\
\text { institute of ethics } \\
(2005) \text { modified by } \\
\text { Mu'in (2011) and } \\
\text { Gould (2018) }\end{array}$} & \multicolumn{2}{|c|}{ Techniques } \\
\hline & & & & & $\begin{array}{l}\text { Expli } \\
\text { citly }\end{array}$ & $\begin{array}{l}\text { Impli } \\
\text { citly }\end{array}$ \\
\hline \multirow[t]{5}{*}{1} & \multicolumn{6}{|c|}{ Data in SPTLP/Syllabus } \\
\hline & \multirow[t]{4}{*}{$\begin{array}{l}\text { Universi } \\
\text { ty A }\end{array}$} & & $\begin{array}{l}\text { A./A/1./ } \\
1 / \mathrm{a}\end{array}$ & $\mathrm{R} 3 \mathrm{a}$ & $\begin{array}{l}\text { Expli } \\
\text { citly }\end{array}$ & \\
\hline & & & $\begin{array}{l}\text { A./A/2./ } \\
1 / \mathrm{a}\end{array}$ & $\begin{array}{l}\mathrm{R} 2 \mathrm{a} \\
\mathrm{C} 5 \mathrm{~b}\end{array}$ & $\begin{array}{l}\text { Expli } \\
\text { citly }\end{array}$ & \\
\hline & & & $\begin{array}{l}\text { A./A/2./ } \\
1 / \mathrm{b}\end{array}$ & $\begin{array}{l}\mathrm{R} 3 \mathrm{a} \\
\mathrm{C} 5 \mathrm{~b}\end{array}$ & $\begin{array}{l}\text { Expli } \\
\text { citly }\end{array}$ & \\
\hline & & & $\begin{array}{l}\text { A./A/2./ } \\
1 / \mathrm{c}\end{array}$ & $\begin{array}{l}\mathrm{R} 2 \mathrm{a} \\
\mathrm{C} 5 \mathrm{~b}\end{array}$ & $\begin{array}{l}\text { Expli } \\
\text { citly }\end{array}$ & \\
\hline
\end{tabular}




\begin{tabular}{|c|c|c|c|c|c|c|}
\hline & & \begin{tabular}{|l}
$\mathrm{A} . / \mathrm{A} / 2 . /$ \\
$1 / \mathrm{d}$
\end{tabular} & $\mathrm{R} 2 \mathrm{a}$ & $\begin{array}{c}\text { Expli } \\
\text { citly }\end{array}$ & \\
\hline & & & $\begin{array}{l}\text { A./A/2./ } \\
1 / \mathrm{e}\end{array}$ & $\mathrm{R} 3 \mathrm{a}$ & $\begin{array}{l}\text { Expli } \\
\text { citly }\end{array}$ & \\
\hline & & & $\begin{array}{l}\mathrm{A} . / \mathrm{A} / 3 . / \\
1 / \mathrm{a} \\
\end{array}$ & T1a & $\begin{array}{l}\text { Expli } \\
\text { citly }\end{array}$ & \\
\hline & & & $\begin{array}{l}\text { A./A/4./ } \\
1 / \mathrm{a} \\
\end{array}$ & C6a & & $\begin{array}{l}\text { Impli } \\
\text { citly }\end{array}$ \\
\hline & & & \begin{tabular}{|l} 
A./A/4./ \\
$1 / \mathrm{b}$
\end{tabular} & $\mathrm{T} 1 \mathrm{~b}$ & $\begin{array}{l}\text { Expli } \\
\text { citly }\end{array}$ & \\
\hline & & & $\begin{array}{l}\mathrm{A} . / \mathrm{A} / 5 . / \\
1 / \mathrm{a} \\
\end{array}$ & $\begin{array}{l}\text { T1a } \\
\text { R2a } \\
\end{array}$ & $\begin{array}{l}\text { Expli } \\
\text { citly }\end{array}$ & \\
\hline & & & \begin{tabular}{|l} 
A./A/6./ \\
$1 / \mathrm{a}$
\end{tabular} & R3a & $\begin{array}{l}\text { Expli } \\
\text { citly }\end{array}$ & \\
\hline & \multirow[t]{9}{*}{$\begin{array}{l}\text { Universi } \\
\text { ty B }\end{array}$} & & \begin{tabular}{|l|}
$\mathrm{B} . / \mathrm{A} / 1 . /$ \\
$2 / \mathrm{a}$
\end{tabular} & $\mathrm{R} 3 \mathrm{a}$ & $\begin{array}{l}\text { Expli } \\
\text { citly }\end{array}$ & \\
\hline & & & \begin{tabular}{|l|}
$\mathrm{B} . / \mathrm{A} / 2 . /$ \\
$2 / \mathrm{a}$
\end{tabular} & $\mathrm{R} 2 \mathrm{c}$ & $\begin{array}{l}\text { Expli } \\
\text { citly }\end{array}$ & \\
\hline & & & \begin{tabular}{|l|}
$\mathrm{B} . / \mathrm{A} / 2 . /$ \\
$2 / \mathrm{b}$
\end{tabular} & $\begin{array}{l}\mathrm{C} 5 \mathrm{a} \\
\mathrm{C} 5 \mathrm{~b}\end{array}$ & $\begin{array}{l}\text { Expli } \\
\text { citly }\end{array}$ & \\
\hline & & & \begin{tabular}{|l|}
$\mathrm{B} . / \mathrm{A} / 3 . /$ \\
$2 / \mathrm{a}$
\end{tabular} & $\mathrm{R} 3 \mathrm{a}$ & & $\begin{array}{l}\text { Impli } \\
\text { citly }\end{array}$ \\
\hline & & & \begin{tabular}{|l|}
$\mathrm{B} . / \mathrm{A} / 4 . /$ \\
$2 / \mathrm{a}$
\end{tabular} & R3a & $\begin{array}{l}\text { Expli } \\
\text { citly }\end{array}$ & \\
\hline & & & \begin{tabular}{|l} 
B./A/4./ \\
$2 / \mathrm{b}$
\end{tabular} & R3a & $\begin{array}{l}\text { Expli } \\
\text { citly }\end{array}$ & \\
\hline & & & \begin{tabular}{|l|}
$\mathrm{B} . / \mathrm{A} / 5 . /$ \\
$2 / \mathrm{a}$
\end{tabular} & $\begin{array}{c}\text { C6d } \\
\text { C5a/b }\end{array}$ & $\begin{array}{l}\text { Expli } \\
\text { citly }\end{array}$ & \\
\hline & & & \begin{tabular}{|l}
$\mathrm{B} . / \mathrm{A} / 5 . /$ \\
$2 / \mathrm{b}$
\end{tabular} & $\begin{array}{l}\text { R3b } \\
\text { T1a }\end{array}$ & $\begin{array}{l}\text { Expli } \\
\text { citly }\end{array}$ & \\
\hline & & & \begin{tabular}{|l}
$\mathrm{B} . / \mathrm{A} / 5 . /$ \\
$2 / \mathrm{c}$ \\
\end{tabular} & R3a & $\begin{array}{l}\text { Expli } \\
\text { citly }\end{array}$ & \\
\hline & \multirow[t]{7}{*}{$\begin{array}{l}\text { Universi } \\
\text { ty } \mathrm{C}\end{array}$} & & \begin{tabular}{|l|}
$\mathrm{C} . / \mathrm{A} / 1 . /$ \\
$3 / \mathrm{a}$
\end{tabular} & $\mathrm{R} 2 \mathrm{c}$ & $\begin{array}{l}\text { Expli } \\
\text { citly }\end{array}$ & \\
\hline & & & \begin{tabular}{|l|}
$\mathrm{C} . / \mathrm{A} / 2 . /$ \\
$3 / \mathrm{a}$
\end{tabular} & $\mathrm{R} 2 \mathrm{a}$ & $\begin{array}{l}\text { Expli } \\
\text { citly }\end{array}$ & \\
\hline & & & \begin{tabular}{|l|}
$\mathrm{C} . / \mathrm{A} / 2 . /$ \\
$3 / \mathrm{b}$
\end{tabular} & $\mathrm{R} 3 \mathrm{a}$ & $\begin{array}{l}\text { Expli } \\
\text { citly }\end{array}$ & \\
\hline & & & \begin{tabular}{|l|}
$\mathrm{C} . / \mathrm{A} / 3 . /$ \\
$1 / \mathrm{a}$
\end{tabular} & $\mathrm{T} 1 \mathrm{~b}$ & $\begin{array}{l}\text { Expli } \\
\text { citly }\end{array}$ & \\
\hline & & & $\begin{array}{l}\mathrm{C} . / \mathrm{A} / 3 . / \\
1 / \mathrm{b} \\
\end{array}$ & $\mathrm{C} 5 \mathrm{~b}$ & $\begin{array}{l}\text { Expli } \\
\text { citly }\end{array}$ & \\
\hline & & & $\begin{array}{l}\text { C./A/4./ } \\
1 / \mathrm{a}\end{array}$ & $\mathrm{R} 3 \mathrm{a}$ & $\begin{array}{l}\text { Expli } \\
\text { citly }\end{array}$ & \\
\hline & & & \begin{tabular}{|l|}
$\mathrm{C} . / \mathrm{A} / 5 . /$ \\
3/a
\end{tabular} & C6a & $\begin{array}{l}\text { Expli } \\
\text { citly }\end{array}$ & \\
\hline 2 & \multicolumn{6}{|c|}{ Data in Learning Materials } \\
\hline
\end{tabular}




\begin{tabular}{|c|c|c|c|c|}
\hline \multirow[t]{13}{*}{$\begin{array}{l}\text { Universi } \\
\text { ty A }\end{array}$} & $\begin{array}{l}\mathrm{A} . / \mathrm{B} / 2 . / \\
1 / \mathrm{a}\end{array}$ & $\mathrm{T} 1 \mathrm{a}$ & & $\begin{array}{l}\text { Impli } \\
\text { citly }\end{array}$ \\
\hline & $\begin{array}{l}\text { A./B/2./ } \\
1 / \mathrm{b}\end{array}$ & $\mathrm{R} 2 \mathrm{a}$ & & $\begin{array}{l}\text { Impli } \\
\text { citly }\end{array}$ \\
\hline & $\begin{array}{l}\mathrm{A} . / \mathrm{B} / 2 . / \\
1 / \mathrm{c}\end{array}$ & $\mathrm{R} 2 \mathrm{a}$ & & $\begin{array}{l}\text { Impli } \\
\text { citly }\end{array}$ \\
\hline & $\begin{array}{l}\mathrm{A} . / \mathrm{B} / 2 . / \\
1 / \mathrm{d}\end{array}$ & $\mathrm{C} 5 \mathrm{~b}$ & & $\begin{array}{l}\text { Impli } \\
\text { citly }\end{array}$ \\
\hline & $\begin{array}{l}\mathrm{A} . / \mathrm{B} / 2 . / \\
1 / \mathrm{e}\end{array}$ & $\mathrm{R} 2 \mathrm{a}$ & & $\begin{array}{l}\text { Impli } \\
\text { citly }\end{array}$ \\
\hline & $\begin{array}{l}\text { A./B/2./ } \\
1 / \mathrm{f}\end{array}$ & $\mathrm{R} 2 \mathrm{a}$ & & $\begin{array}{l}\text { Impli } \\
\text { citly }\end{array}$ \\
\hline & $\begin{array}{l}\text { A./B/2./ } \\
1 / \mathrm{g}\end{array}$ & $\mathrm{C} 5 \mathrm{~b}$ & & $\begin{array}{l}\text { Impli } \\
\text { citly }\end{array}$ \\
\hline & $\begin{array}{l}\text { A./B/2.I } \\
1 / \mathrm{h}\end{array}$ & $\mathrm{R} 2 \mathrm{a}$ & & $\begin{array}{l}\text { Impli } \\
\text { citly }\end{array}$ \\
\hline & $\begin{array}{l}\text { A./B/4./ } \\
1 / \mathrm{a}\end{array}$ & $\mathrm{R} 2 \mathrm{a}$ & & $\begin{array}{l}\text { Impli } \\
\text { citly }\end{array}$ \\
\hline & $\begin{array}{l}\text { A./B/4./ } \\
1 / \mathrm{b}\end{array}$ & T1a & & $\begin{array}{l}\text { Impli } \\
\text { citly }\end{array}$ \\
\hline & $\begin{array}{l}\text { A./B/4./ } \\
1 / \mathrm{c}\end{array}$ & $\mathrm{R} 3 \mathrm{a}$ & & $\begin{array}{l}\text { Impli } \\
\text { citly }\end{array}$ \\
\hline & $\begin{array}{l}\text { A./B/5./ } \\
1 / \mathrm{a}\end{array}$ & $\mathrm{C} 5 \mathrm{~b}$ & & $\begin{array}{l}\text { Impli } \\
\text { citly }\end{array}$ \\
\hline & $\begin{array}{l}\mathrm{A} . / \mathrm{B} / 7 . / \\
1 / \mathrm{a}\end{array}$ & $\mathrm{C} 5 \mathrm{~b}$ & & $\begin{array}{l}\text { Impli } \\
\text { citly }\end{array}$ \\
\hline \multirow[t]{9}{*}{$\begin{array}{l}\text { Universi } \\
\text { ty B }\end{array}$} & $\begin{array}{l}\mathrm{B} . / \mathrm{B} / 5 . / \\
2 / \mathrm{a}\end{array}$ & $\mathrm{T} 1 \mathrm{a}$ & & $\begin{array}{l}\text { Impli } \\
\text { citly }\end{array}$ \\
\hline & $\begin{array}{l}\text { B./B/5./ } \\
2 / \mathrm{b}\end{array}$ & $\mathrm{R} 2 \mathrm{a}$ & & $\begin{array}{l}\text { Impli } \\
\text { citly }\end{array}$ \\
\hline & $\begin{array}{l}\mathrm{B} . / \mathrm{B} / 5 . / \\
2 / \mathrm{c}\end{array}$ & $\begin{array}{l}\mathrm{R} 2 \mathrm{a} \\
\mathrm{F} 4 \mathrm{~b}\end{array}$ & & $\begin{array}{l}\text { Impli } \\
\text { citly }\end{array}$ \\
\hline & $\begin{array}{l}\text { B./B/5./ } \\
2 / \mathrm{d}\end{array}$ & $\begin{array}{l}\text { R2a } \\
\text { F4b }\end{array}$ & & $\begin{array}{l}\text { Impli } \\
\text { citly }\end{array}$ \\
\hline & $\begin{array}{l}\mathrm{B} . / \mathrm{B} / 5 . / \\
2 / \mathrm{e}\end{array}$ & $\begin{array}{l}\mathrm{R} 2 \mathrm{a} \\
\mathrm{F} 4 \mathrm{~b} \\
\end{array}$ & & $\begin{array}{l}\text { Impli } \\
\text { citly }\end{array}$ \\
\hline & $\begin{array}{l}\text { B./B/5./ } \\
2 / \mathrm{f}\end{array}$ & $\begin{array}{l}\mathrm{R} 2 \mathrm{a} \\
\mathrm{C} 5 \mathrm{~b}\end{array}$ & & $\begin{array}{l}\text { Impli } \\
\text { citly }\end{array}$ \\
\hline & $\begin{array}{l}\mathrm{B} . / \mathrm{B} / 5 . / \\
2 / \mathrm{g}\end{array}$ & $\mathrm{F} 4 \mathrm{~b}$ & & $\begin{array}{l}\text { Impli } \\
\text { citly }\end{array}$ \\
\hline & $\begin{array}{l}\text { B./B/6./ } \\
2 / \mathrm{a}\end{array}$ & $\mathrm{T} 1 \mathrm{a}$ & & $\begin{array}{l}\text { Impli } \\
\text { citly }\end{array}$ \\
\hline & $\begin{array}{l}\text { B./B/6./ } \\
2 / \mathrm{b}\end{array}$ & T1a & & $\begin{array}{l}\text { Impli } \\
\text { citly }\end{array}$ \\
\hline \multirow[t]{2}{*}{$\begin{array}{l}\text { Universi } \\
\text { ty C }\end{array}$} & $\begin{array}{l}\text { C./B/2./ } \\
1 / \mathrm{a}\end{array}$ & $\mathrm{R} 3 \mathrm{a}$ & $\begin{array}{l}\text { Expli } \\
\text { citly }\end{array}$ & \\
\hline & C./B/6./ & $\mathrm{R} 3 \mathrm{~b}$ & & Impli \\
\hline
\end{tabular}




\begin{tabular}{|c|c|c|c|c|c|}
\hline & & 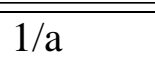 & 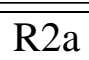 & & " citly \\
\hline \multirow{12}{*}{\multicolumn{2}{|c|}{$\begin{array}{l}\text { Data in A } \\
\text { Universi } \\
\text { ty A }\end{array}$}} & \multicolumn{4}{|c|}{ Data in Assessments } \\
\hline & & $\begin{array}{l}\mathrm{A} . / \mathrm{C} / 2 . / \\
1 / \mathrm{a}\end{array}$ & $\begin{array}{l}\text { T1a } \\
\text { R3a }\end{array}$ & & $\begin{array}{l}\text { Impli } \\
\text { citly }\end{array}$ \\
\hline & & $\begin{array}{l}\text { A./C/2./ } \\
1 / \mathrm{b}\end{array}$ & $\begin{array}{l}\text { T1a } \\
\text { R3a }\end{array}$ & & $\begin{array}{l}\text { Impli } \\
\text { citly }\end{array}$ \\
\hline & & $\begin{array}{l}\text { A./C/2./ } \\
1 / \mathrm{c}\end{array}$ & $\begin{array}{l}\text { T1a } \\
\text { R3a }\end{array}$ & & $\begin{array}{l}\text { Impli } \\
\text { citly }\end{array}$ \\
\hline & & $\begin{array}{l}\mathrm{A} . / \mathrm{C} / 2 . / \\
1 / \mathrm{d}\end{array}$ & $\mathrm{R} 3 \mathrm{a}$ & & $\begin{array}{l}\text { Impli } \\
\text { citly }\end{array}$ \\
\hline & & $\begin{array}{l}\mathrm{B} . / \mathrm{C} / 1 . / \\
2 / \mathrm{a}\end{array}$ & $\begin{array}{l}\text { R2a } \\
\text { R3b }\end{array}$ & & $\begin{array}{l}\text { Impli } \\
\text { citly }\end{array}$ \\
\hline & & $\begin{array}{l}\text { B./C/2./ } \\
2 / \mathrm{a}\end{array}$ & $\mathrm{R} 3 \mathrm{c}$ & & $\begin{array}{l}\text { Impli } \\
\text { citly }\end{array}$ \\
\hline & & $\begin{array}{l}\text { B./C/3./ } \\
2 / \mathrm{a}\end{array}$ & $\mathrm{R} 3 \mathrm{a}$ & & $\begin{array}{l}\text { Impli } \\
\text { citly }\end{array}$ \\
\hline & & $\begin{array}{l}\text { B./C/3./ } \\
2 / b\end{array}$ & $\mathrm{R} 3 \mathrm{a}$ & & $\begin{array}{l}\text { Impli } \\
\text { citly }\end{array}$ \\
\hline & & $\begin{array}{l}\text { B./C/4./ } \\
2 / \mathrm{a}\end{array}$ & R3a & & $\begin{array}{l}\text { Impli } \\
\text { citly }\end{array}$ \\
\hline & & $\begin{array}{l}\text { B./C/5./ } \\
2 / \mathrm{a}\end{array}$ & $\mathrm{R} 3 \mathrm{a}$ & & $\begin{array}{l}\text { Impli } \\
\text { citly }\end{array}$ \\
\hline & & $\begin{array}{l}\text { B./C/7./ } \\
\text { 2/a }\end{array}$ & R3a & & $\begin{array}{l}\text { Impli } \\
\text { citly }\end{array}$ \\
\hline & $\begin{array}{l}\text { Universi } \\
\text { ty } \mathrm{C}\end{array}$ & $\begin{array}{l}\mathrm{C} . / \mathrm{C} / 1 . / \\
3 / \mathrm{a}\end{array}$ & $\mathrm{R} 3 \mathrm{a}$ & $\begin{array}{l}\text { Expli } \\
\text { citly }\end{array}$ & \\
\hline & & $\begin{array}{l}\mathrm{C} . / \mathrm{C} / 1 . / \\
3 / \mathrm{b}\end{array}$ & $\mathrm{R} 3 \mathrm{a}$ & $\begin{array}{l}\text { Expli } \\
\text { citly }\end{array}$ & \\
\hline & & $\begin{array}{l}\text { C./C/1./ } \\
3 / \mathrm{c}\end{array}$ & R3a & $\begin{array}{l}\text { Expli } \\
\text { citly }\end{array}$ & \\
\hline & & $\begin{array}{l}\mathrm{C} . / \mathrm{C} / 2 . / \\
1 / \mathrm{a}\end{array}$ & $\mathrm{R} 3 \mathrm{a}$ & $\begin{array}{l}\text { Expli } \\
\text { citly }\end{array}$ & \\
\hline & & $\begin{array}{l}\mathrm{C} . / \mathrm{C} / 2 . / \\
1 / \mathrm{b}\end{array}$ & $\mathrm{T} 1 \mathrm{~b}$ & $\begin{array}{l}\text { Expli } \\
\text { citly }\end{array}$ & \\
\hline & & $\begin{array}{l}\text { C./C/5./ } \\
3 / \mathrm{a}\end{array}$ & $\mathrm{R} 3 \mathrm{a}$ & $\begin{array}{l}\text { Expli } \\
\text { citly }\end{array}$ & \\
\hline
\end{tabular}

Adopted and modified from (Sulistianingrum, 2015)

The findings of character building of ethics addressed in English curriculum documents - semester planning of teaching and learning program (SPTLP)/syllabus, learning materials, and assessments which cover the meaning of core ethical values. Then, they are described by providing the examples from the data. The core of ethical values consist of trustworthiness, respect, responsibility, fairness, caring, and citizenship (Josephson, 2005). This ethical values were modified by Mu'in (2011) and Gould (2018)

For the discussion of related to addressing ethics in curriculum documents, the relevant study was conducted by (Hidayat, 2013) about aklhlakul karimah which 
refers to pedoman penskoran akhlak mulia. It consists responsibility, discipline, confidence, competitive, politeness, social relationship, honesty, worship, cleanness, and health. One of the ten values mentions honesty can measure someone's behavior. Hence, this character is very important to be available in shaping students' attitudes.

The example of ethical values addressed in University A such as beriman dan berakhlak mulia (A./A/3./1/a) in syllabus, greeting (A./B/2./1/b), mentaati (A./A/1./1/a), menawarkan pertolongan (A./A/2./1/a), mengembangkan diri sebagai masyarakat umum dalam mengembangkan bahasa Inggris (A./A/4./1/a). These words represented the ethical values of trustworthiness, respect, responsibility, caring and citizenship.

In University $\mathrm{B}$, the words addressing ethical values were like akhlak mulia (B.A/5./2/b), menghargai keanekaragaman budaya, agama, pandangan, kepercayaan, pendapat, dan temuan orang lain (B./A/2./2/a), menunjukan sikap bertanggung jawab (B.A/1./2/a), membrikan informasi mengenai jdwal konsultasi atau pemeriksaan kesehatan (B./B/5./2/c), peduli terhadap masyarakat dan lingkungan (B./A/5./2/a), and bekerja sama serta memiliki kepekaan sosial terhadap masyarakat dan lingkungan (B./A/5./2/a). These words covered the ethical values of trustworthiness, respect, responsibility, fairness, caring, and citizenship.

University $\mathrm{C}$ addressed the ethical values of trustworthiness, respect, responsibility, caring, and citizenship. For examples iman dan takwa (C./A/3./1/a), respect of character building, students are not allowed to chit-chat during learning sessions and are expected to seriously and activity take part of learning activities, respect one's opinions, and help each other (C./A/1./3/a), melaksanakan perkuliahan sesuai SAP dan tatatertib (C./A/4./1/a), membimbing peserta didik mengembangkan keterampilan social (C./A/3./1/b), and Students are expected to begin developing the required personality traits of a professional Indonesian EFL educator. Professional quality is expected to be seen in all aspects of their work, academic and social interactions with other class members, as well as in their personal appearance.

From the result of discussion, ethical values were addressed in building students' character in university's documents and six core ethical values - trustworthiness, respect, responsibility, fairness, caring, and citizenship - were presented in SPTLP/syllabus, learning material, and assessment. The forms of data addressing are words, phrases, and clauses which covers the ethical values.

The addressing of ethical values in the documents encourage students to speak and behave good things in order to have good values because this is so important to be shaped so that it will become a habit which will be beneficial for students' future. Therefore, these good values will cause not only to the individual person but to each institution also.

\section{CONCLUSION}

Finally, this study shows the consistency of addressing character building of ethic in undergraduate English curriculum document. From the conclusion, each university has its own way in addressing ethical values in each curriculum document. University A was consistent in addressing ethical values of Trustworthiness and Responsibility, University B was consistent with ethical values of Respect, and University C was consistent with ethical values of Responsibility. The consistencies are that character building of ethics is presented in semester planning of teaching and learning program (SPTLP)/syllabus, learning material, and assessment. 


\section{REFERENCES}

Acetylena, S. (2013). Analisis Implementasi Kebijakan Pendidikan Karakter Di Perguruan Taman Siswa Kecamatan Turen Kabupaten Malang. Jurnal Kebijakan Dan Pengembangan Pendidikan, 1(1), 56-61. Retrieved from http://ejournal.umm.ac.id/index.php/jmkpp/article/viewFile/1509/1610

Butts, J. B. (2001). Ethics in professional nursing practice. JOGN Nursing; Journal of Obstetric, Gynecologic, and Neonatal Nursing, 8(6), 327-329. Retrieved from http://www.ncbi.nlm.nih.gov/pubmed/18524302

Furman, G. C. (2004). The ethic of community. Journal of Educational Administration, 42(2), 215-235. https://doi.org/10.1108/09578230410525612

Gould, J. (2018). Universal Core Ethical Values. cardea

Hidayat, A. (2013). Persepsi dan Perilaku Mahasiswa dalam Pendidikan Karakter, 1(June 2010), 2014-2015.

Huberman, M. (1994). Qualitative Data Analysis.

Isyraqi, A. (2011). Etika Sosial Persaudaraan dan Perdamaian ( Studi di Malaysia dan Indonesia ), 5, 39-54.

Jact, R., and Fraenkel, N.E.W. (2009). How to Design and Evaluate Research In Education.

Josephson institute of ethics. (2005). Making Ethical Decisions. Communications of the ACM, 43(12), 66-71. https://doi.org/10.1145/355112.355126

Jovanovic, S., and Wood, R. V. (2006). Communication ethics and ethical culture: A study of the ethics initiative in Denver City government. Journal of Applied Communication Research, 34(4), 386-405. https://doi.org/10.1080/00909880600908633

Margetson, D. (1997). Ethics in assessing and developing academic quality. Assessment and Evaluation in Higher Education, 22(2), 123-133. https://doi.org/10.1080/0260293970220203

Mohammad, C. (2016). Emphasizing Morals, Values, Ethics, and Character Education in Science Education and Science Teaching., 4(2), 1-16.

Mu'in, F. (2011). Pendidikan Karakter-Konstruksi Teoritik \& Praktik-Urgensi Pendidikan Progresif dan Revitalisasi Peran Guru dan Orang Tua. yogyakarta: ArRuzz Media.

Nijhof, A., Wilderom, C., and Oost, M. (2012). Professional and institutional morality: building ethics programmes on the dual loyalty of academic professionals. Ethics and Education, 7(1), 91-109. https://doi.org/10.1080/17449642.2012.681236

Popoola, I. "Tobi," Garner, B., Ammeter, A., Krey, N., Beu Ammeter, D., \& Schafer, S. (2017). How does ethics institutionalization reduce academic cheating? Journal of Education for Business, 92(1), 29-35. https://doi.org/10.1080/08832323.2016.1274710

Safatly, L., Itani, H., El-Hajj, A., and Salem, D. (2017). Ethics centers' activities and role in promoting ethics in universities. Ethics and Education, 12(2), 153-169. https://doi.org/10.1080/17449642.2017.1286787

Spears, M. C. (2006). Academic ethics [4]. Current Science, 91(5), 567. https://doi.org/10.1057/omj.2008.7

Sulistiyaningrum, F. (2015). An Analysis Of Characters In The Texts Of An English Textbook Entitled Contextual English For Grade Xi Of Senior High Schools Regular Program.

Syamsiyatun, S., dan Wafiroh, N. (2013). Filsafat, Etika, dan Kearifan Lokal. 